\section{FIRST-IN-HUMAN RESULTS WITH THE NOVEL TUMOR- TARGETING ANTIBODY ATRC-101: PHASE 1B STUDY IN PATIENTS WITH SOLID TUMORS}

1John Powderly*, ${ }^{2}$ Jeremy Jones, ${ }^{2}$ Tanios Bekaii-Saab, ${ }^{3}$ Yan Xing, ${ }^{2} \mathrm{~S}$ John Weroha, ${ }^{4}$ Susanna Ulahannan, ${ }^{5}$ Deborah Doroshow, ${ }^{6}$ Frances Valdes-Albini, ${ }^{7}$ Carl Millward ${ }^{7}$ Kimberly Walter, ${ }^{7}$ Andrew Wrong, ${ }^{7}$ Paul Del Castillo, ${ }^{7}$ Lixia Wang, ${ }^{7}$ Ngan Nguyen, ${ }^{7}$ Mark Whidden, ${ }^{7}$ Jonathan Benjamin, ${ }^{8}$ Steven Isakoff. ${ }^{1}$ Carolina BioOncology Institute, Huntersville, NC, USA; ${ }^{2}$ Mayo Clinic, Jacksonville, FL, USA; ${ }^{3}$ City of Hope Comprehensive Cancer Center, Duarte, CA, USA; ${ }^{4}$ University of Oklahoma Health Stephenson Cancer Center, OKC, OK, USA; ${ }^{5}$ Mount Sinai, New York, NY, USA; ${ }^{6}$ University of Miami Sylvester Comprehensive Cancer Center, Miami, FL, USA; ${ }^{7}$ Atreca, Inc, San Carlos, CA, USA;

${ }^{8}$ Massachusetts General Hospital, Boston, MA, USA

Background ATRC-101 is an engineered version of an immunoglobulin G1 antibody that was discovered in a patient with non-small cell lung cancer (NSCLC) experiencing stable disease while being treated with anti-programmed death-1 therapy. ATRC-101 targets a tumor-specific ribonucleoprotein complex containing polyadenylate binding protein-1, which has been found to be present in the majority of NSCLC, acral melanoma, breast, colorectal, and ovarian cancer samples tested. Target immunoreactivity and single-agent activity have been observed in mouse models. Preclinical data suggest that ATRC-101 stimulates both innate and adaptive immune activity against tumors.

Methods ATRC-101-A01 is a phase $1 \mathrm{~b}$ trial $(3+3$ dose escalation with expansion cohorts) in patients with solid tumors treated with ATRC-101 monotherapy every 2 or 3 weeks (Q2W or Q3W), or ATRC-101 in combination with pembrolizumab, until unacceptable toxicity or disease progression at doses of $0.3-30 \mathrm{mg} / \mathrm{kg}$, pending dose-limiting toxicities. The primary objective is safety and secondary objectives are to characterize the pharmacokinetic profile, immunogenicity, and anti-tumor activity of ATRC-101, and to determine the recommended dose for expansion. Pharmacodynamic studies will also be performed to evaluate changes from baseline in specific immune cell populations and cytokine levels in blood and tumors. Results from the ATRC-101 $0.3-30 \mathrm{mg} / \mathrm{kg}$ monotherapy Q3W cohorts are presented in this abstract (data cutoff: July 16, 2021).

Results Twenty-four participants with solid tumors (13 colorectal, 5 ovarian, 3 breast, 2 NSCLC, 1 acral melanoma) aged 27-75 years with a median 5 lines of prior therapy were treated Q3W in five dose cohorts. No dose-limiting toxicities were observed. Eight participants (33\%) experienced grade $\geq 3$ treatment-emergent adverse events. The maximum serum concentration of ATRC-101 and treatment exposure appeared to be dose proportional. Stable disease was observed in eight patients and best response per RECIST v1.1 was associated with expression of the ATRC-101 target. Multiple biomarkers, such as treatment-associated changes in the composition of $\mathrm{CD} 3+, \mathrm{CD} 4+$, and CD8 $+\mathrm{T}$ cells in the blood, and serum cytokines/chemokines, including those predicted to activate antigen-presentation pathways, support the proposed mechanism of action of ATRC-101 and will be presented.

Conclusions These first-in-human data suggest a manageable safety profile for ATRC-101 Q3W, with no dose-limiting toxicities observed. Pharmacokinetics appear to be dose proportional. Enrollment in the Q2W monotherapy dose-escalation cohort and at the $30 \mathrm{mg} / \mathrm{kg}$ dose level Q3W is continuing. Trial sites have been activated to test ATRC-101 in combination with pembrolizumab, and combination with chemotherapy is also planned.

Trial Registration Trial Registration: NCT04244552
Ethics Approval This study was approved by the institutional review board or ethics committee as required for each participating site.

http://dx.doi.org/10.1136/jitc-2021-SITC2021.518 